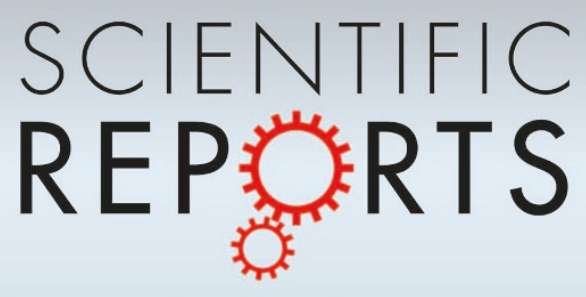

OPEN

SUBJECT AREAS:

SEMICONDUCTORS

ELECTRONIC PROPERTIES AND

MATERIALS

ELECTRONIC STRUCTURE

Received

11 September 2013

Accepted

26 November 2013

Published

12 December 2013

Correspondence and requests for materials should be addressed to

R.A.L. (roger@uow. edu.au)

\section{Spherical, cylindrical and tetrahedral symmetries; hydrogenic states at high magnetic field in Si:P}

\author{
R. A. Lewis' ${ }^{1}$ A. Bruno-Alfonso ${ }^{2}$, G. V. B. de Souza ${ }^{3}$, R. E. M. Vickers' ${ }^{1}$ J. A. Colla' \& E. Constable'
}

'Institute for Superconducting and Electronic Materials, University of Wollongong, Wollongong, New South Wales 2522, Australia, ${ }^{2}$ Faculdade de Ciências, UNESP-Universidade Estadual Paulista, 17033-360, Bauru, SP, Brazil, ${ }^{3}$ POSMAT/UNESP-Universidade Estadual Paulista, 17033-360, Bauru, SP, Brazil.

Phosphorous donors in silicon have an electronic structure that mimics the hydrogen atom, albeit on a larger length, smaller energy and smaller magnetic field scale. While the hydrogen atom is spherically symmetric, an applied magnetic field imposes cylindrical symmetry, and the solid-state analogue involves, in addition, the symmetry of the Si crystal. For one magnetic field direction, all six conduction-band valleys of Si:P become equivalent. New experimental data to high laboratory fields $(30 \mathrm{~T})$, supported by new calculations, demonstrate that this high symmetry field orientation allows the most direct comparison with free hydrogen.

T he recent work of Murdin et al. ${ }^{1}$ impressively demonstrates that an impurity atom in a semiconductor host acts as a hydrogen atom in a white dwarf star, yet in a laboratory magnetic field about one thousand times less than the stellar field. In free hydrogen, the space between the proton and the electron is a vacuum. In the laboratory analogue, the introduced $\mathrm{P}^{+}$atom and the donated $\mathrm{e}^{-}$are immersed in a crystal of Si. The effective mass, $m^{*}$, of the electron is decreased and the relative permittivity, $\epsilon_{\mathrm{r}}$, of the medium is increased with respect to the vacuum values of $m_{\mathrm{e}}$ and 1 . Both changes work to increase the orbital radius, $a^{*}=\left(\epsilon_{\mathrm{r}} m_{\mathrm{e}} / m^{*}\right) a_{0}$, from the Bohr radius $a_{0}$, to decrease the binding energy, $R^{*}=\left(m^{*} / m_{\mathrm{e}} \epsilon_{\mathrm{r}}^{2}\right) R$, from the Rydberg $R$, and to reduce the characteristic magnetic field, $B_{0}^{*}=m^{* 2} e^{3} / 2 \epsilon_{\mathrm{r}}^{2} \kappa^{2} \hbar^{3}$, from the value for the hydrogen atom (where $e, \kappa$ and $\hbar$ are the electron charge, Coulomb constant, and reduced Planck constant, respectively). Importantly, the crystal matrix introduces another factor-a reduction in symmetry. The previous work was limited to magnetic fields along the $\langle 100\rangle$ crystallographic direction. Varying the crystal direction relative to the magnetic field gives an additional control. Another magnetic field orientation, $\mathbf{B} \|\langle 111\rangle$, is noteworthy. This is the unique orientation for which all six conduction-band valleys make the same angle with the magnetic field. Consequently, the six conduction-band valleys are all equivalent. It follows that the energy levels, and the allowed transitions, are simpler than for the $\langle 100\rangle$ orientation. Here we present data for $\mathbf{B}||\langle 111\rangle$ in Si:P to an equally high field (30 T), supported by new calculations, simultaneously comprehensively confirming and extending the previous work and allowing a simpler comparison with the hydrogen atom, as the fewer transitions involved are easier to identify.

\section{Results}

As Murdin et al. ${ }^{1}$ point out, the hydrogenic Lyman spectra are simple both at low field, where the magnetic field can be treated as a perturbation, and at high field, where the Coulomb field can be treated as a perturbation. The most interesting, complex and challenging region is the intermediate field and we will focus our attention on this. Figure 1 shows the Lyman spectrum for Si:P that we have calculated (full lines) and measured (circled dots). We will now compare these new results both to the previous calculations of the hydrogen spectrum (Fig. 3 of ref. 1) and to the previous calculations and experiments for the Si:P spectrum (Fig. 2 of ref. 1). We note our results are in good agreement with those of $\mathrm{Mu}$ et al. ${ }^{2}$ as far as that work extends, to $10 \mathrm{~T}$.

With respect to the hydrogen spectrum, Fig. 3 of ref. 1, we note that the exact solution has been given previously by Kravchenko et al. ${ }^{3}$ with numerical values listed for the states $1 s, 2 s, 2 p_{0}, 2 p_{-}, 3 p_{0}, 3 p_{-}, 3 d_{-}$and $3 d_{2-}$. We have calculated the states of hydrogen using a finite-difference method ${ }^{4}$ and now using a Rayleigh-Ritz method and in each case obtain excellent agreement with the values given in ref. 3. Turning now to the Si:P spectrum, the exact method of solution cannot be applied due to the complication of crystalline anisotropy. The finite-difference 


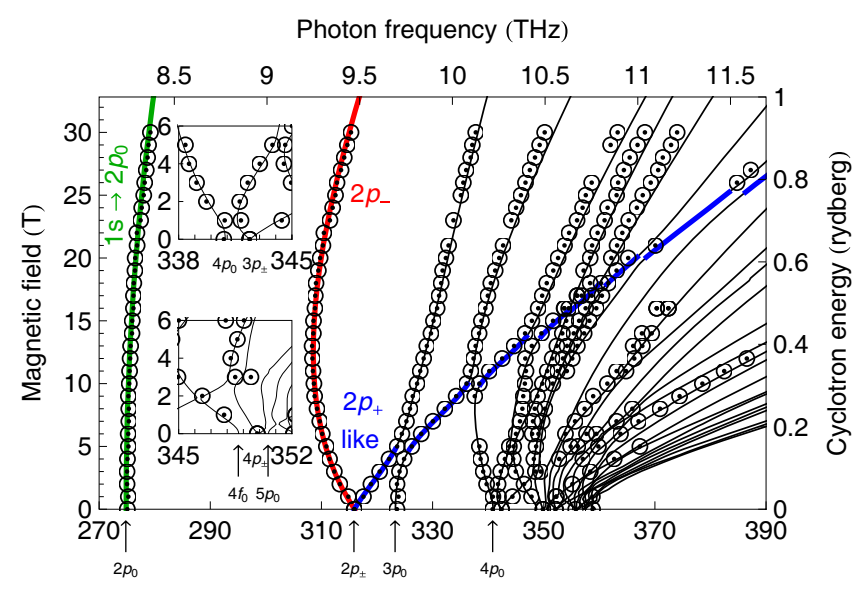

Photon spatial frequency $\left(\mathrm{cm}^{-1}\right)$

Figure $1 \mid$ Calculated (lines) and observed (circled dots) frequencies of Lyman transitions for Si:P as a function of magnetic field B for $B||\langle 111\rangle$.

method is still applicable, but involves large matrices ${ }^{4}$. Instead, we employ a variational method ${ }^{5}$, which uses much smaller matrices and so is much more efficient. Since our previous work $^{5}$ we have improved the minimisation. Details are given in the Methods section below. The Lanczos procedure of ref. 1 represents another calculational approach.

We divide our discussion into two parts, separated by the prominent $2 p_{+}$transition, which runs diagonally from the bottom middle to the top right corner of Fig. 1. We first consider lower-energy transitions, that is, ones lying to the left of the $2 p_{+}$transition in Fig. 1. In the hydrogen spectrum (Fig. 3 of ref. 1), along with $2 p_{+}$, the transitions originating from the zero-field $n=2$ transition (at $\frac{3}{4} R^{*}$ ) are $2 p_{-}$and $2 p_{0}$. (The $2 s$ state also originates here, but transitions are forbidden from the ground state $1 s$ to the $2 s$ state, so it is not displayed.) The other transitions in this energy regime are the pairs $3 p_{-} / 3 p_{0}, 4 p_{-} / 4 p_{0}$, and $5 p_{-} / 5 p_{0}$, all shown (but not labelled) on Fig. 3 of ref. 1. Compare this with the case of Si:P, B ||$\langle 100\rangle$, Fig. 2 of ref. 1. A conspicuous difference is that the crystal symmetry lifts the degeneracy of the $n=2$ states; the state labelled $2 p_{\text {ox }}$ has a different energy

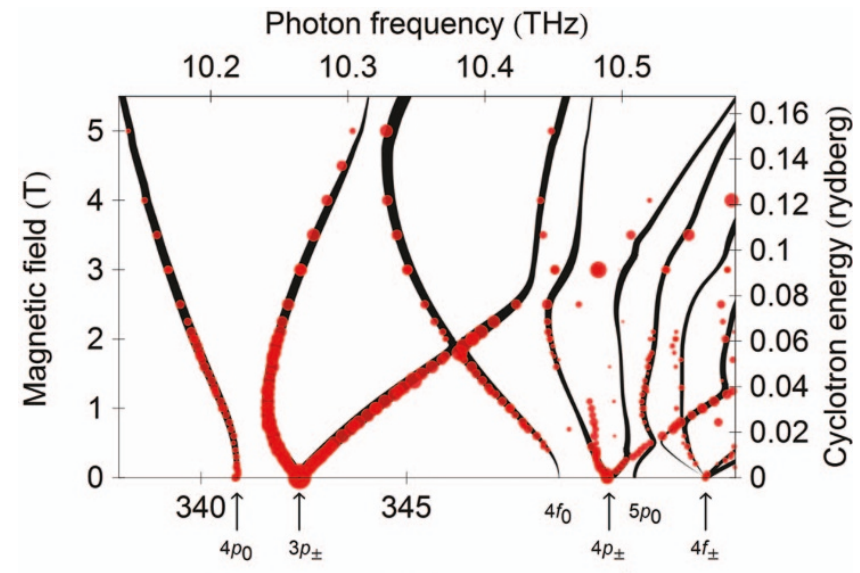

Photon spatial frequency $\left(\mathrm{cm}^{-1}\right)$

Figure $2 \mid$ Calculated and observed frequencies and strengths of Lyman transitions for Si:P as a function of magnetic field B for B ||$\langle 111\rangle$ in the region of high energy and low magnetic field. Circles represent the experimental data; the radii are proportional to the area of the absorption peaks. Lines represent the theoretical data; the widths of the lines are proportional to the square root of the calculated absorption strengths.
Photon frequency $(\mathrm{THz})$

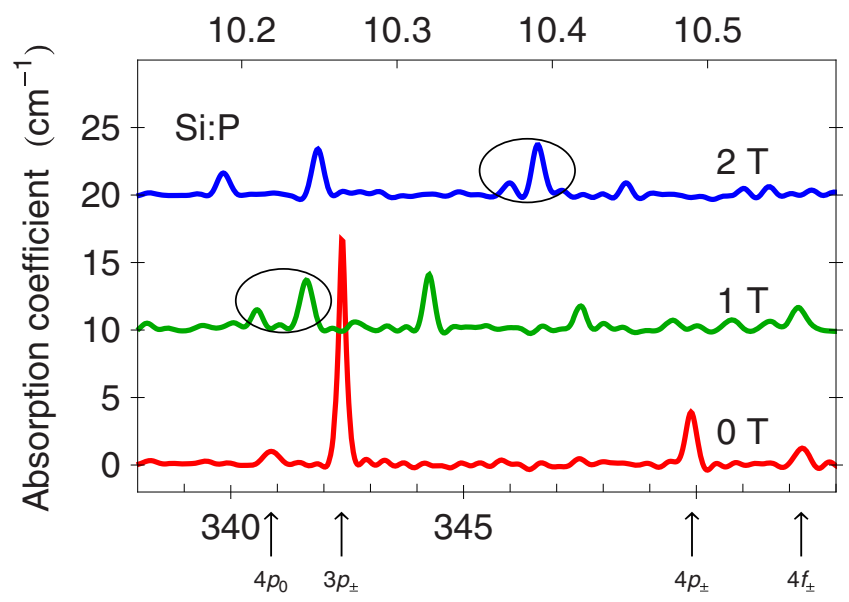

Photon spatial frequency $\left(\mathrm{cm}^{-1}\right)$

Figure $3 \mid$ Experimental spectra of Si:P at magnetic fields of $B=0,1$ and $2 \mathrm{~T}$ with $\mathrm{B}||\langle\mathbf{1 1 1}\rangle$. The absorption coefficient has been calculated in the usual way. For clarity, the spectra for 1 and $2 \mathrm{~T}$ have been offset on the vertical axis by 10 and $20 \mathrm{~cm}^{-1}$, respectively. As highlighted, the lower branch of the transition labelled $3 p_{ \pm}$interacts with the transition labelled $4 p_{0}$ at around $1 \mathrm{~T}$ and the upper branch of transition labelled $3 p_{ \pm}$interacts with the transition labelled $4 f_{0}$ (not evident at $0 \mathrm{~T}$ ) at around $2 \mathrm{~T}$.

to the $2 p_{ \pm}$states, even in the absence of magnetic field. As well, the region between the $2 p_{-}$and $3 p_{-}$transitions no longer has a lone transition $\left(2 p_{0}\right)$, but four transitions now appear. The high-energy region at the highest magnetic fields is even more cluttered, with some ten additional unpolarised transitions in addition to the clear $3 p_{-}, 4 p_{-}$and $5 p_{-}$. Finally, compare with the case of Si:P, B ||$\langle 111\rangle$ shown in Fig. 1. The split-off $2 p_{0}$ state is evident, well separated from the others, as for $\mathbf{B}||\langle 100\rangle$. However, in contrast to $\mathbf{B} \|\langle 100\rangle$, the region between the $2 p_{-}$and $3 p_{-}$is much less cluttered, containing only the $3 p_{0}$ state. Moreover, in the high-energy, high-field region, there are far fewer transitions expected and observed and there is an excellent agreement between experiment and calculation.

Turning now to the $2 p_{+}$transition itself, in contrast to $\mathbf{B} \|\langle 100\rangle$, the $\mathbf{B} \|\langle 111\rangle$ orientation yields repeated examples of anti-crossing. The first three anti-crossings are clear in the experimental data. The theoretical absorption intensities calculated as function of magnetic field reveal seven anti-crossings within the field range 0-30 T.

Finally, to better deal with transitions of higher energies than the $2 p_{+}$transition, we will focus on the low-field region 0-6 T (Fig. 2, Fig. 3). Both the positions and the intensities of the observed and calculated transitions are shown. In comparison to Fig. 5 of ref. 1, at the lowest energies and fields we distinctly observe the $4 p_{0}$ transition. The data for that transition, and the repulsion of the adjacent $3 p_{-}$ transition, are very distinct and in excellent agreement with the calculations. In the same way, the data for the $3 p_{-}$transition follows the calculation very precisely, with a second anti-crossing evident at about $5 \mathrm{~T}$. The simpler crystallographic orientation means the whole region is less cluttered than for $\mathbf{B} \| \mid\langle 100\rangle$; in particular, the most intense transitions of Fig. 5 of ref. 1, occurring between the $3 p_{ \pm}$ and $4 p_{ \pm}$zero-field energies, do not appear at all for $\mathbf{B} \|\langle 111\rangle$, leaving a striking example of a crossing at around $2 \mathrm{~T}$ in the middle of this energy range. Further such detailed observations may be made; however, this becomes progressively more difficult at higher energies due to the diminishing intensities and increasing number of the transitions.

To conclude, the general problem of the semiconductor analogue of free hydrogen in a magnetic field involves three symmetries: the 
crystal tetrahedral symmetry interacting with the magnetic field cylindrical symmetry and the electric (Coulombic) spherical symmetry. We have made measurements and calculations to magnetic fields at which the cyclotron energy approaches the binding energy with the field along the simplest crystal direction. Clear-cut agreement is found between the data and model, increasing confidence in the extension of such laboratory analogues to astrophysical phenomena.

\section{Methods}

Experimental methods. The sample used was a single crystal of silicon doped by neutron transmutation to a phosphorous concentration of $\sim 1 \times 10^{14} \mathrm{~cm}^{-3}$. The residual concentration of other donors and acceptors was estimated to be below $10^{12} \mathrm{~cm}^{-3}$; no transitions other than from Si:P were observed in the spectra. The sample was wedged to suppress interference fringes. Data to $30 \mathrm{~T}$ with the sample at $4.2 \mathrm{~K}$ were collected using a Bruker 113v Fourier spectrometer operating at unapodised resolution of $1 \mathrm{~cm}^{-1}(0.03 \mathrm{THz}, 0.12 \mathrm{meV})$. Data to $6 \mathrm{~T}$ with the sample at $10 \mathrm{~K}$ were collected using a modified Polytec Fourier spectrometer operating at unapodised resolution of $0.4 \mathrm{~cm}^{-1}(0.01 \mathrm{THz}, 0.05 \mathrm{meV})$. In both cases, a liquidhelium-cooled Si-composite bolometer was used as the detector.

Theoretical methods. The effective-mass equation for the donor impurity in silicon is solved for a single valley of the conduction band, and the symmetric gauge is chosen for the magnetic field. With the field along the [111] direction, the six valleys are equivalent and we disregard both the coupling between them and the central-cell correction. The latter is expected to be relevant for the ground $1 s$ state only. Therefore, the dependence of the ground energy level on the magnetic field strength is corrected by fitting the experimental data for $1 s \rightarrow 2 p_{0}$ transition energies. As a result, the $1 s$ level is shifted by $\Delta E=-115.45-0.00225 B^{2}$, with $B$ given in Tesla. For the calculation of the dipole transition matrix elements, however, we retain the uncorrected $1 s$ wavefunction.

Because of the inversion symmetry, the donor wavefunctions are symmetric or antisymmetric. The ground state belongs to the first class, while the excited states of the dipole-allowed transitions are of the second class. The donor states are calculated by the Rayleigh-Ritz method ${ }^{6}$. Each state is given by a linear combination of elongated, scaled and orthonormal hydrogenic orbitals of the following form ${ }^{2,5}$ :

$$
\xi_{l, m}^{3 / 2} \beta^{1 / 2} \psi_{n, l, m}\left[\xi_{l, m}(x, y, \beta z)\right],
$$

where $n, l$ and $m$ are the atomic quantum numbers. The elongation is given by the variational parameter $\beta$, which is expected to take into account the anisotropy induced by the effective mass and the magnetic field, while the scaling variational parameter $\xi_{l, m}$ is intended to adjust the size of the basis function for the combined effect of the Coulomb and magnetic fields. The symmetry of the basis functions is chosen to agree with the symmetry of the donor state. The Hamiltonian matrix elements are calculated analytically ${ }^{2}$. Hence, for each symmetry and for given values of the elongation and scaling variational parameters, a simple diagonalisation technique produces a set of estimates for the donor energies and the corresponding coefficients of the linear combinations. Moreover, each donor state may be improved separately, by minimising the corresponding energy as a function of the elongation and scaling variational parameters.

For each symmetry, the calculations have been performed by including the states with $n \leq 6$. This leads to a set of 47 (44) basis functions for the symmetric (antisymmetric) donor states, and the energy minimisation is performed over the elongation parameter $\beta$ and the 21 (15) scaling variational parameters. The relatively large number of variational parameters gives some flexibility to the trial wavefunctions. Accordingly, the energy of each level may present multiple local minima. Since iterative minimisation procedures are used, several choices of the starting points are made in order to reach the lowest local minimum. The simplest of such choices is the set of variational parameters that minimises the trace of the Hamiltonian matrix ${ }^{5,6}$.

After the energy levels and the parameters of the variational trial functions have been obtained as a function of the magnetic-field strength, the $1 s \rightarrow$ (antisymmetric state) transition energies and the corresponding absorption strengths are determined. For unpolarised radiation, the latter is directly proportional to the square of the matrix element of the electron position times the transition energy ${ }^{7}$.

1. Murdin, B. N. et al. Si:P as a laboratory analogue for hydrogen on high magnetic field white dwarf stars. Nat. Commun. 4, 1469; DOI:10.1038/ncomms2466 (2013).

2. Mu, Y.-M. et al. Effects of an external magnetic field on shallow donor levels in semiconductors. Phys. Rev. B 48, 10864-10869 (1993).

3. Kravchenko, Yu. P., Liberman, M. A. \& Johansson, B. Exact solution for hydrogen atom in a magnetic field of arbitrary strength. Phys. Rev. A 54, 287-305 (1996).

4. de Souza, G. V. B. \& Bruno-Alfonso, A. Bound hydrogenic states in a magnetic field: a finite-difference approach. Int. J. Mod. Phys. B 23, 3009-3013 (2009).

5. Bruno-Alfonso, A., Lewis, R. A., Hai, G. Q. \& Vickers, R. E. M. Energy states of phosphorous donor in silicon in fields up to 18 T. J. Low Temp. Phys. 159, 226-229 (2010).

6. Kościk, P. \& Okopińska, A. The optimized Rayleigh-Ritz scheme for determining the quantum-mechanical spectrum. J. Phys. A: Math. Theor. 40, 10851-10869 (2007).

7. Shen, S.-C., Zhu, J.-B., Mu, Y.-M. \& Liu, P.-L. Wave-function mixture and composition for hybridized Zeeman states of P in Si. Phys. Rev. B 49, 5300-5305 (1994).

\section{Acknowledgments}

This work was supported by the Australian Research Council and the University of Wollongong. A part of the work was performed at the National High Magnetic Field Laboratory, which is supported by NSF Cooperative Agreement No. DMR-9527035 and by the State of Florida. We are grateful to the Brazilian Agencies CNPq (including a fellowship under Science Without Borders) and FAPESP, as well as to the Graduate Program POSMAT/Unesp, for financial support.

\section{Author contributions}

R.A.L. and A.B.-A. designed the research programme. R.A.L., R.E.M.V., J.A.C. and E.C. performed the experiments. A.B.-A. and G.V.B.d.S. carried out the calculations.

\section{Additional information}

Competing financial interests: The authors declare no competing financial interests.

How to cite this article: Lewis, R.A. et al. Spherical, cylindrical and tetrahedral symmetries; hydrogenic states at high magnetic field in Si:P. Sci. Rep. 3, 3488; DOI:10.1038/srep03488 (2013).

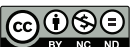

This work is licensed under a Creative Commons AttributionNonCommercial-NoDerivs 3.0 Unported license. To view a copy of this license, visit http://creativecommons.org/licenses/by-nc-nd/3.0 